\title{
As várias faces da rinite
}

\author{
RevPort Imunoalergologia 202I;29 (2):8I-83
}

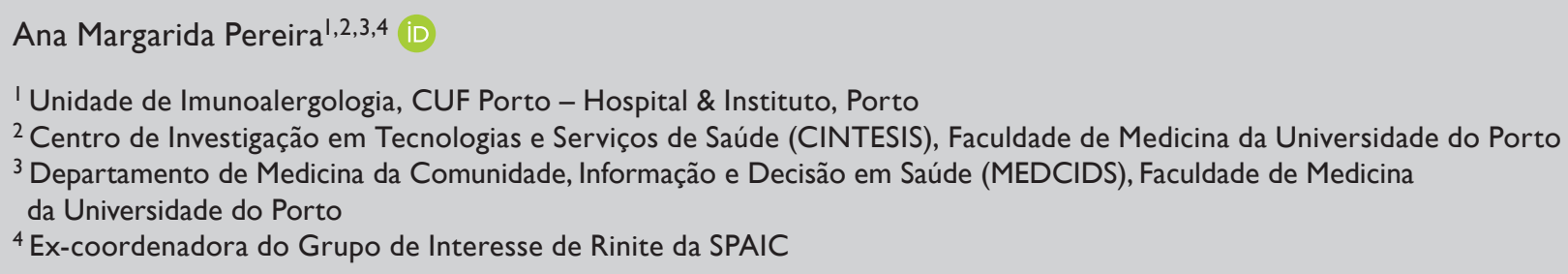

A rinite é uma das patologias médicas mais comuns $^{1,2}$ e, provavelmente, a que vemos mais frequentemente na consulta de Imunoalergologia. Carateriza-se pela presença de sintomas nasais, como congestão/obstrução nasal, rinorreia, prurido nasal e espirros, causados por inflamação e/ou disfunção a nível da mucosa do nariz',3. Estes sintomas ocorrem pelo menos dois dias seguidos e durante mais de uma hora na maioria dos dias $\mathbf{1}^{1,4,5}$. Apesar de ser uma patologia frequentemente desvalorizada (quer pelos doentes, quer pelos médicos), a rinite associa-se a perda de qualidade de vida, com impacto no sono, na capacidade de trabalho e no rendimento escolar ${ }^{4,6}$. Tem custos diretos e indiretos elevados ${ }^{4,7}$.

A relevância da rinite, especialmente pela sua prevalência e impacto na vida dos doentes, torna fundamental um adequado reconhecimento e abordagem, sendo central o papel do imunoalergologista. Um dos aspetos diferenciadores é a capacidade de reconhecimento da rinite como uma patologia heterogénea, que tem diversas etiologias, várias formas de apresentação clínica e de resposta ao tratamento ${ }^{1,3}$. Nesse sentido, neste número da RPIA damos início a uma série de artigos, na Página
Educacional da revista, que irão abordar, numa perspetiva prática, várias das faces da rinite.

A heterogeneidade da rinite levou à necessidade de a classificar, tendo surgido, especialmente nas últimas 2 décadas, múltiplas propostas de classificação baseadas em diferentes aspetos da doença' ${ }^{1,3,4}$. Existem classificações que distinguem as diferentes formas de rinite com base nos mecanismos patofisiológicos que lhes estão subjacentes, identificando endótipos ${ }^{3,8,9}$. As classificações endotípicas, apesar de muito relevantes, são ainda limitadas e nem sempre passíveis de avaliação adequada com os meios de diagnóstico disponíveis na prática clínica diária9. As classificações mais frequentes e mais usadas clinicamente baseiam-se na identificação de fenótipos, que agrupam os indivíduos de acordo com caraterísticas clínicas observáveis (traits) associadas à rinite ${ }^{1,3}$. Os fenótipos podem ser definidos considerando diversos critérios clínicos (por exemplo, idade de início, gravidade, sazonalidade, frequência dos sintomas ou os seus desencadeantes), estando descritos múltiplos sistemas de classificação (exemplificados no Quadro I) com maior ou menor relevância do ponto de vista clínico. A maioria destas classificações fenotípicas foi de- 
senvolvida unicamente com base em hipóteses a priori, a partir do conhecimento teórico disponível sobre a rinite, sendo ainda relativamente escassos os estudos que descrevem fenótipos obtidos a partir de técnicas estatísticas de classificação em clusters ${ }^{10,11}$, ao contrário do que já acontece na asma ${ }^{12}$. Estes fenótipos "clássicos", que não são estabelecidos diretamente a partir dos dados de doentes com rinite, são dinâmicos e podem sobrepor-se, dificultando definições claras ${ }^{3}$. Em 2015, numa tentativa de uniformizar a classificação fenotípica dos indivíduos com rinite, foi proposta pelo PRACTALL ${ }^{3}$ uma classificação por consenso em cinco fenótipos: rinite infeciosa, rinossinusite crónica, rinite alérgica, rinite alérgica local e rinite não alérgica. Apesar de nenhuma destas classificações ser universalmente aceite nem usada de forma exclusiva, elas são potencialmente relevantes na personalização dos cuidados prestados aos doentes com rinite ${ }^{3,8}$ e são parte integrante da nossa prática clínica diária.

Os fenótipos que serão abordados nesta série de artigos são baseados na classificação proposta pelo PRACTALL ${ }^{3}$, incluindo, de forma independente, a rinite ocupacional e a rinite mista. Os dois primeiros artigos, publicados neste número da RPIA, focam a rinite alérgica e a rinite ocupacional. Estes artigos seguem a estrutura dos manuscritos da secção Clinical Practice, do New England Journal of Medicine $^{13}$, em que o tema é lançado usando um caso clínico muito sumário (Case Vignette), que foca um dos fenótipos "clássicos" de rinite e serve de ponto de partida para uma revisão da evidência sobre essa situação clínica, abordando diferentes aspetos que se consideram clinicamente relevantes (por exemplo, caraterísticas clínicas, diagnóstico e tratamento). $\mathrm{O}$ artigo termina com as recomendações clínicas dos autores, tendo por base o caso clínico inicial.

Os casos clínicos que serão incluídos nos vários artigos foram propostos por internos de Imunoalergologia no contexto de um pedido de colaboração do Grupo de Interesse de Rinite para preparação de um workshop que decorreu na 40. ${ }^{\text {a }}$ Reunião Anual da SPAIC, em 2019 (Workshop III subordinado ao tema "Rinite: uma família de patologias. Casos clínicos"). Os internos que propuseram casos clínicos foram desafiados a elaborar estes artigos, sendo que só a sua disponibilidade e empenho permitiram que eles se concretizassem.

Esperamos que esta série de artigos ajude a estruturar a abordagem clínica das diferentes formas de rinite e que possa ser usada pelos imunoalergologistas mais jovens (e também pelos menos jovens!) na melhoria da qualidade dos cuidados prestados aos doentes com rinite.

\section{Conflito de interesses}

O autor declara que não existem conflitos de interesses.

Quadro I. Exemplos de classificações fenotípicas da rinite ${ }^{\mathrm{l}, 3}$

\begin{tabular}{|l|l|}
\hline Classificação baseada em: & \multicolumn{1}{c|}{ Fenótipos } \\
\hline Gravidade & Ligeira / moderada-grave / severe chronic upper airway disease \\
\hline Padrão temporal & Sazonal / perene \\
\hline Duração dos sintomas & Aguda ou crónica / intermitente ou persistente \\
\hline Sintoma predominante & Runners / blockers \\
\hline Controlo & Controlado / não controlado \\
\hline Desencadeante & Alergénio / infeção / fármaco / ... \\
\hline Consenso PRACTALL & $\begin{array}{l}\text { Rinite infeciosa / rinossinusite crónica / rinite alérgica / rinite alérgica local / rinite não alérgica } \\
\text { (inclui rinite idiopática, hormonal, gustativa, induzida por fármacos, atrófica, ocupacional e do idoso) }\end{array}$ \\
\hline
\end{tabular}




\section{ORCID:}

Ana Margarida Pereira (iD 0000-0002-5468-0932

\section{REFERÊNCIAS}

I. Mullol J, Del Cuvillo A, Lockey RF. Rhinitis Phenotypes. J Allergy Clin Immunol Pract 2020;8(5): I492-503.

2. Bousquet J, Anto JM, Bachert C, Baiardini I, Bosnic-Anticevich S, Walter Canonica G, et al. Allergic rhinitis. Nat Rev Dis Primers 2020;6(I):95.

3. Papadopoulos NG, Bernstein JA, Demoly P, Dykewicz M, Fokkens $W$, Hellings $P W$, et al. Phenotypes and endotypes of rhinitis and their impact on management: a PRACTALL report. Allergy 2015;70(5):474-94.

4. Bousquet J, Khaltaev N, Cruz A, Denburg J, Fokkens W, Togias A, et al. Allergic Rhinitis and its Impact on Asthma (ARIA) 2008. Allergy 2008;63(8):160-9.

5. International Rhinitis Management Working G. International consensus report on the diagnosis and management of allergic rhinitis. Allergy 1994;49:5-34.

6. Meltzer EO, Blaiss MS, Derebery MJ, Mahr TA, Gordon BR, Sheth $\mathrm{KK}$, et al. Burden of allergic rhinitis: results from the pediatric allergies in America survey. J Allergy Clin Immunol 2009;124 (3 Suppl):S43-70.

7. Avdeeva KS, Reitsma S, Fokkens WJ. Direct and indirect costs of allergic and non-allergic rhinitis in the Netherlands. Allergy 2020;75(II):2993-6.

8. Hellings PW, Klimek L, Cingi C, Agache I, Akdis C, Bachert C, et al. Non-allergic rhinitis: Position paper of the European Academy of Allergy and Clinical Immunology. Allergy 20I7;72(II):1657-65.

9. Meng Y, Lou H, Wang Y, Wang X, Cao F, Wang K, et al. Endotypes of chronic rhinitis: A cluster analysis study. Allergy 2019;74(4):720-30.

10. Kurukulaaratchy RJ, Zhang H, Patil V, Raza A, Karmaus W, Ewart $\mathrm{S}$, et al. Identifying the heterogeneity of young adult rhinitis through cluster analysis in the Isle of Wight birth cohort. J Allergy Clin Immunol 2015;135(I):143-50.e7.

II. Burte E, Bousquet J, Varraso R, Gormand F, Just J, Matran R, et al. Characterization of rhinitis according to the asthma status in adults using an unsupervised approach in the EGEA study. PLoS One 2015;10(8):e0136191.

12. Cunha F, Amaral R, Jacinto T, Sousa-Pinto B, Fonseca JA. A Systematic review of asthma phenotypes derived by data-driven methods. diagnostics (Basileia) 2021;II(4):644.

13. Hopkins C. Chronic Rhinosinusitis with nasal polyps. N Engl J Med 2019;38I(I):55-63. 